\title{
Bandwidth expansion of a pressure control system for pneumatic anti-vibration apparatuses in presence of dead time
}

\author{
Yukinori NAKAMURA*, Hiroki KAWAKAMI** and Shinji WAKUI* \\ * Institute of Engineering, Tokyo University of Agriculture and Technology \\ 2-24-16 Naka, Koganei, Tokyo 184-8588, Japan \\ E-mail: yukino-n@cc.tuat.ac.jp \\ ** Faculty of Engineering, Tokyo University of Agriculture and Technology \\ 2-24-16 Naka, Koganei, Tokyo 184-8588, Japan
}

Received 15 March 2015

\begin{abstract}
This paper considers the pressure control of air springs used for a pneumatic anti-vibration apparatus (AVA), which isolates vibration transmitted to semiconductor lithography equipment. In the control of the pneumatic AVA, pressure feedback is often employed so as to keep the inner pressure of air springs constant. By expanding the bandwidth of pressure control system, fast response can be obtained, though this bandwidth is restricted due to anti-resonance and resonance in a pneumatic system. To eliminate the anti-resonance and resonance, relative displacement derivative (RDD) positive feedback control has been presented. However, previous RDD positive feedback control cannot be implemented under the condition where the dead time of air springs exists. In order to compensate effects of the dead time, a Smith predictor is utilized. Through experiments, it is shown that in the presence of the dead time, the anti-resonance and resonance can be eliminated by means of the RDD positive feedback control combined with Smith prediction. The bandwidth of the pressure control system increases with the gain of pressure PI compensator. Furthermore, effects of proposed approach on vibration transmissibility are investigated. Although the vibration transmissibility is the performance metric for the development of the pneumatic AVA, the case where the dead time is present was not sufficiently considered. In this paper, it is shown that the vibration transmissibility changes in accordance with the dead time.
\end{abstract}

Key words : Pneumatic equipment, Vibration control, Vibration isolation, Pressure control, Dead time

\section{Introduction}

A pneumatic anti-vibration apparatus (AVA) (Ryaboy, 2014; Heertjes et al., 2013; Bulter, 2011; Pu et al., 2011; Shin et al., 2010) plays a crucial role in the manufacturing process of integrated circuits. By using this apparatus, it is possible to reduce effects of the vibration transmitted to the XY stage in lithography equipment. Air springs of the pneumatic AVA support the isolation table where the XY stage is mounted. The driving force of the air springs is regulated by servo valves used for the exhaust and intake of compressed air. However, since the values of parameters of each air spring are different, generated driving forces are also different. For this reason, the structure in the lithography equipment is deformed. Moreover, since the XY stage moves on the isolation table during lithography process, the position of the pneumatic AVA's center of gravity changes. Consequently, the isolation table is inclined. The structure deformation of lithography equipment and the inclination of isolation table cause the large positioning error and long settling time of the XY stage (Wakui and Fushima, 2006).

One approach for the above two problems is to use pressure feedback control (Shin and Kim, 2009; Wakui, 2003; Canon Inc., 2001a). This feedback control is implemented in the commercial pneumatic AVA so as to avoid the structure deformation of lithography equipment. In addition to the pressure feedback control, when the pressure feedforward 
control (Shin and Kim, 2009; Canon Inc., 2001b) is also used, the inclination of the isolation table is rapidly corrected. Although the fast response of a pressure control system can be achieved by expanding its bandwidth, it is necessary to consider anti-resonance and resonance in a pneumatic system, which corresponds to the air springs. When the gain of pressure compensator increases so as to expand the bandwidth, the resonance peak becomes large. This causes the destabilization of the control system. Thus, it is difficult to expand the control bandwidth of the pressure control system under the condition that the anti-resonance and resonance exist.

Wakui and Fushima (2006) proposed the relative displacement derivative (RDD) positive feedback control. This control scheme uses the information on the derivative of the relative displacement between the isolation table and the floor. Due to the feedback of the above information, the anti-resonance and resonance can be eliminated. However, in Wakui and Fushima (2006), the dead time of the air spring (i.e., the time delay in the response of the pneumatic system) was not considered. In the industry of semiconductor manufacturing, a large pneumatic AVA is needed so as to support heavy lithography equipment. For this reason, it takes time to inspect the servo valve attached to each air spring. To avoid this difficulty, all servo valves are gathered and set apart from the air springs (Nakamura et al., 2013). Consequently, the pipe connecting the servo valve and the air spring becomes long, resulting in the increase of the dead time.

As mentioned in subsection 3.1, when the dead time exists, it is necessary to know a priori information on the displacement derivative of the isolation table so as to use the RDD positive feedback control. In other words, the future output of a displacement sensor has to be obtained. For this reason, the system with the RDD positive feedback control is not causal in the presence of the dead time, and the control bandwidth of pressure control system cannot be expanded. Shin and Kim (2009) presented the feedback and feedforward control schemes which use the inner pressure of air springs. Kawashima et al. (2007) developed pressure differentiator in order to decrease the natural frequency of the pneumatic AVA. However, these works did not address the problem of the bandwidth expansion of pressure control system with the dead time. Thus, the dead time of the air spring is a practical issue that should be solved.

In this paper, we consider how to expand the bandwidth of the pressure control system with the dead time of the air spring. The first contribution of this paper is to present the method for the elimination of the anti-resonance and resonance. To reduce effects of the dead time, a Smith predictor (Smith, 1959) is employed. By using the RDD positive feedback combined with the Smith prediction, the anti-resonance and resonance can be eliminated even if the dead time exists. Furthermore, the bandwidth of the pressure control system can be expanded by tuning the gain of a pressure PI compensator. Experiments demonstrate that a well-known Smith predictor approach used for dead time compensation is effective in terms of the bandwidth expansion of pressure control system.

The second contribution of this paper is to investigate effects of our proposed method on vibration transmissibility. In the development of the pneumatic AVA and lithography equipment, the vibration transmissibility is necessarily employed as a performance metric (e.g, ASML Inc., 2014; Nikon Corp., 2011; Ryaboy, 2014). Although our previous work (Nakamura et al., 2013) considered the isolation of the vibration caused by the dead time, the vibration transmissibility was not discussed. We show that the vibration transmissibility changes in accordance with the dead time.

The rest of the paper is organized as follows. Section 2 explains the pneumatic AVA, the anti-resonance and resonance, and the RDD positive feedback control. Section 3 describes the drawback of the previous RDD positive feedback control and presents the proposed method for the bandwidth expansion of pressure control system with the dead time. Section 4 shows effects of the dead time on the vibration transmissibility. Finally, conclusions are drawn in Section 5.

\section{Pressure control of pneumatic anti-vibration apparatus}

\subsection{Pneumatic anti-vibration apparatus and its control system}

In this subsection, we first explain the pneumatic AVA and its control system. Figure 1 shows the photograph of the pneumatic AVA that is considered in this paper, and Fig. 2 illustrates the experimental setup. The AVA has the air spring which sustains the isolation table. The air spring and compressor are connected with a pipe. Through this pipe, the air generated from the compressor is supplied to the air spring. Since this AVA is an active type isolator, the amount of the air is dynamically adjusted by using the feedback control. Due to the adjustment, the vibration of the isolation table can be attenuated. A pressure sensor (Setra, 204) is employed for the control of the pressure in the air spring. Moreover, a displacement sensor (SDL, NS2300/A) is attached to the AVA in order to measure the relative displacement between the isolation table and the floor. An acceleration sensor (JAE, JA-5V) is also attached so as to measure the acceleration of the isolation table. The output signals of these sensors are fed back to a digital signal processor (DSP), where control law is implemented. In accordance with the control law, the input voltage to the servo valve is decided. 


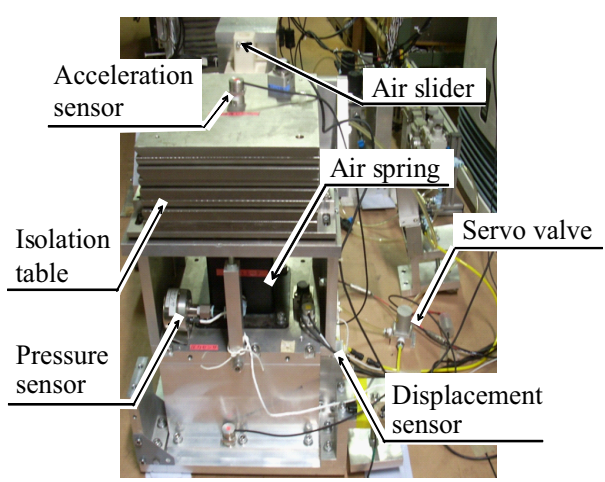

Fig. 1 Pneumatic anti-vibration apparatus. The actuator of this apparatus is a pneumatic spring, whose inner pressure is kept constant by adjusting the amount of the air.

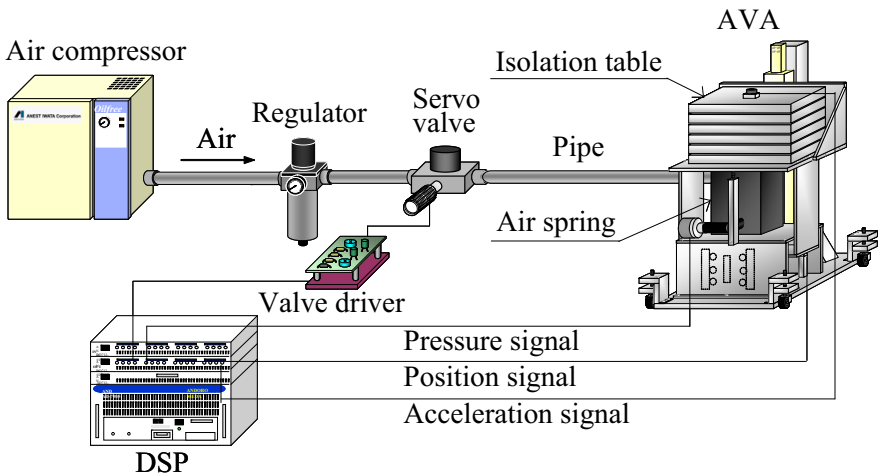

Fig. 2 Experimental setup for pneumatic anti-vibration apparatus. The outputs of pressure, acceleration, and displacement sensors are used as feedback signals in the control system for the pneumatic AVA. The input voltage to the servo valve is determined in accordance with the control law.

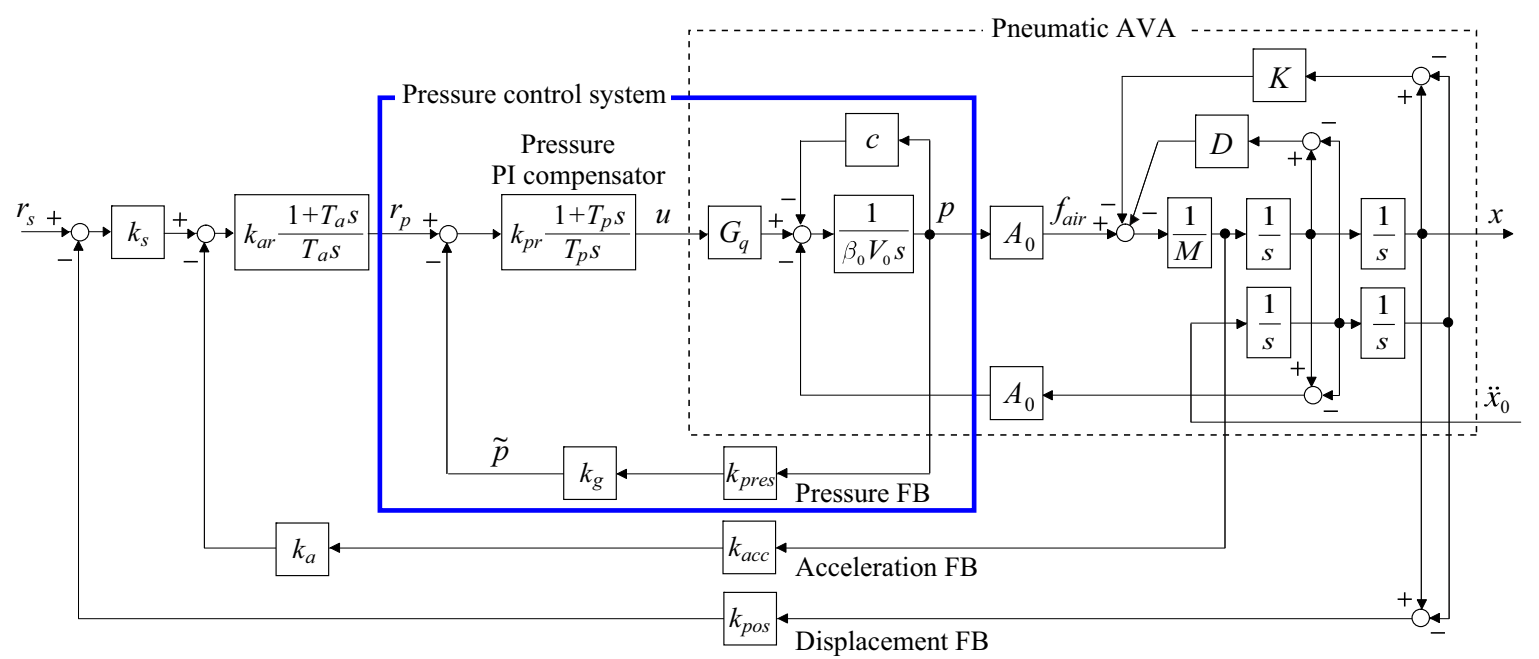

Fig. 3 Block diagram of pneumatic anti-vibration apparatus and its control system. Blocks inside the dashed box show the pneumatic AVA, and blocks inside the blue solid box show the pressure control system. The PI compensator is employed so as to manage the inner pressure of air spring.

Figure 3 shows the block diagram of the pneumatic AVA and its control system. The parameters are summarized in Tables 1 and 2. In Wakui and Fushima (2006), the pneumatic AVA was modeled as a linearized system around equilibrium position of the isolation table. Under the assumption that the displacement $x_{0}$ of the floor is zero, the transfer function $P_{x u}(s)$ from the input voltage $u$ to the displacement $x$ of isolation table is expressed as

$$
P_{x u}(s)=\frac{x(s)}{u(s)}=\frac{1}{M s^{2}+D s+K+\frac{A_{0}^{2} s}{\beta_{0} V_{0} s+c}} \frac{A_{0} G_{q}}{\beta_{0} V_{0} s+c} .
$$

$A_{0} G_{q} /\left(\beta_{0} V_{0} s+c\right)$ in (1) corresponds to the pneumatic system of the AVA, and $M s^{2}+D s+K$ corresponds to the mechanical system of the isolation table.

The control system for the pneumatic AVA consists of displacement, acceleration, and pressure feedback loops. First, the displacement feedback is employed for the levitation of the isolation table. Then, the acceleration feedback control is used to increase the damping of the control system. Finally, to keep the inner pressure of air spring constant, the pressure feedback control is implemented in the control system.

\subsection{Anti-resonance and resonance caused by air spring}

Then, we show that in the pneumatic system of the AVA, there exist anti-resonance and resonance, which prevent the bandwidth expansion of the pressure control system. From Fig. 3, the transfer function $P_{p u}$ from the input voltage $u$ to 
Table 1 Parameters of pneumatic AVA.

\begin{tabular}{c|c|c}
\hline Symbol & Description & Nominal value and unit \\
\hline$M$ & Mass of isolation table & $120.33 \mathrm{~kg}$ \\
$D$ & Viscous damping coefficient & $479 \mathrm{~N} \cdot \mathrm{s} / \mathrm{m}$ \\
$K$ & Spring constant & $11268.4 \mathrm{~N} / \mathrm{m}$ \\
$G_{q}$ & Flow gain & $1.6 \times 10^{-5} \mathrm{~m}^{3} / \mathrm{s} / \mathrm{V}$ \\
$\beta_{0}$ & Compressibility & $2.74 \times 10^{-6} 1 / \mathrm{Pa}$ \\
$c$ & Flow conductance & $4.6 \times 10^{-10} \mathrm{~m}^{3} / \mathrm{s} / \mathrm{Pa}$ \\
$V_{0}$ & Volume of air spring & $1.5 \times 10^{-3} \mathrm{~m}^{3}$ \\
$A_{0}$ & Effective area & $7.8 \times 10^{-3} \mathrm{~m}^{2}$ \\
$u$ & Input voltage to servo valve & $-\mathrm{V}$ \\
$p$ & Inner pressure of air spring & $-\mathrm{Pa}$ \\
$f_{\text {air }}$ & Driving force of air spring & $-\mathrm{N}$ \\
$x$ & Displacement of isolation table & $-\mathrm{m}$ \\
$x_{0}$ & Displacement of floor & $-\mathrm{m}$ \\
\hline
\end{tabular}

the inner pressure $p$ is given by

$$
P_{p u}(s)=\frac{p(s)}{u(s)}=\frac{G_{q}\left(M s^{2}+D s+K\right)}{\left(M s^{2}+D s+K\right)\left(\beta_{0} V_{0} s+c\right)+A_{0}^{2} s} .
$$

Since the flow conductance $c$ is sufficiently small (Wakui and Fushima, 2006), the transfer function (2) is approximated as follows:

$$
P_{p u}(s) \simeq \frac{G_{q}}{\beta_{0} V_{0} s+c} \frac{M s^{2}+D s+K}{M s^{2}+D s+K+K_{a i r}},
$$

where $K_{\text {air }}=A_{0}^{2} /\left(\beta_{0} V_{0}\right)$ denotes the air spring stiffness [N/m] (Lee and Kim, 2007). Note that effects of $M s^{2}+D s+K$ in the numerator of (3) give rise to the anti-resonance. On the other hand, effects of $M s^{2}+D s+K+K_{\text {air }}$ in the denominator of (3) cause the resonance.

\subsection{Effects of RDD positive feedback}

Finally, we describe effects of the RDD positive feedback control (Wakui and Fushima, 2006) on the frequency characteristics of the pneumatic AVA. This control scheme is used to eliminate the anti-resonance and resonance. It leads to the bandwidth expansion of the pressure control system. In the following, we briefly explain this control scheme.

Figure 4 illustrates the block diagram of the RDD positive feedback control. $k_{t u n}$ denotes the tunable parameter of the RDD positive feedback control, and $\tilde{u}$ denotes the output of the pressure PI compensator. From Figs. 3 and 4 , the transfer function from the output $\tilde{u}$ of pressure PI compensator to the inner pressure $p$ of air spring is expressed as

$$
\frac{p(s)}{\tilde{u}(s)}=\frac{G_{q}\left(M s^{2}+D s+K\right)}{\left(M s^{2}+D s+K\right)\left(\beta_{0} V_{0} s+c\right)+A_{0}\left(A_{0}-G_{q} k_{t u n} k_{p o s}\right) s} .
$$

The tunable parameter $k_{t u n}$ is determined so that the anti-resonance and resonance do not exist. By choosing

$$
k_{\text {tun }}=\frac{A_{0}}{G_{q} k_{p o s}}
$$

(4) becomes

$$
\frac{p(s)}{\tilde{u}(s)}=\frac{G_{q}}{\beta_{0} V_{0} s+c} .
$$

Since (6) is a first order lag system, the anti-resonance and resonance of the AVA can be eliminated by means of the RDD positive feedback. Now, by using the air spring stiffness $K_{\text {air }}$, (4) can be approximated as follows:

$$
\frac{p(s)}{\tilde{u}(s)} \simeq \frac{G_{q}}{\beta_{0} V_{0} s+c} \frac{M s^{2}+D s+K}{M s^{2}+D s+K+K_{\text {air }}\left(1-\frac{G_{q} k_{t u n} k_{p o s}}{A_{0}}\right)} .
$$


By substituting (5) into (7), $K_{\text {air }}\left\{1-\left(G_{q} k_{t u n} k_{p o s}\right) / A_{0}\right\}$ in (7) becomes zero. Thus, the physical meaning of the RDD positive feedback control is that effects of the air spring stiffness are cancelled.

Figure 5 shows Bode plots of the transfer function from the output $\tilde{u}$ of pressure PI compensator to the output $k_{\text {pres }} p$ of pressure sensor. In this experiment, a pseudo-differentiator is employed instead of a differentiator shown in Fig. 4 so as to obtain the displacement derivative $\dot{x}$. Moreover, a sinusoidal signal is used as the reference $r_{p}$ of the displacement control system. When the tunable parameter is set as $k_{t u n}=A_{0} /\left(G_{q} k_{p o s}\right)$, the anti-resonance and resonance are suppressed. However, due to the large parameter error of the flow gain $G_{q}$, the anti-resonance and resonance cannot be suppressed in experiment even if nominal value of $G_{q}$ is used for the setting of the tunable parameter $k_{t u n}$. For this reason, we continuously change the value of the tunable parameter $k_{t u n}$ and measure frequency responses. In accordance with the tunable parameter $k_{t u n}$, the anti-resonance and resonance peaks are changed. Furthermore, when the anti-resonance and resonance are suppressed, the phase of the transfer function $p / \tilde{u}$ becomes about $-90 \mathrm{deg}$ in the frequency region higher than break-point frequency. During the continuous change of the tunable parameter, we determine the value of $k_{t u n}$ so that (i) the anti-resonance and resonance peaks are reduced and (ii) the phase becomes about - 90 deg. Due to this method, the tunable parameter is set to $k_{t u n}=23$. The values of parameters used in this experiment are shown in Table 2. From Fig. 5, we can find that the anti-resonance and resonance are eliminated by using the RDD positive feedback control.

From the result of Wakui and Fushima (2006), the closed-loop transfer function of the pressure control system in Fig. 4 is written as

$$
\frac{p(s)}{r_{p}(s)}=\frac{1}{k_{g} k_{\text {pres }}} \frac{1}{\frac{T_{p} c}{G_{q} k_{g} k_{\text {pres }} k_{p r}} s+1}
$$

(8) denotes the transfer function from the reference $r_{p}$ of pressure control system to the inner pressure $p$ of air spring. As the gain $k_{p r}$ of the pressure PI compensator increases, the break-point frequency of (8) becomes high. Thus, one can expand the bandwidth of the pressure control system.

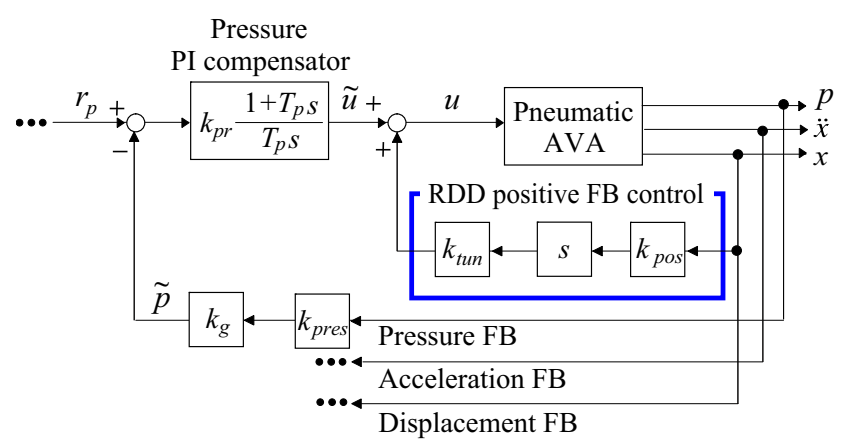

Fig. 4 Block diagram of RDD positive feedback control scheme. The blue solid box contains the RDD positive feedback controller, whose tunable parameter is denoted by $k_{t u n}$. This control scheme is used for the suppression of the anti-resonance and resonance.
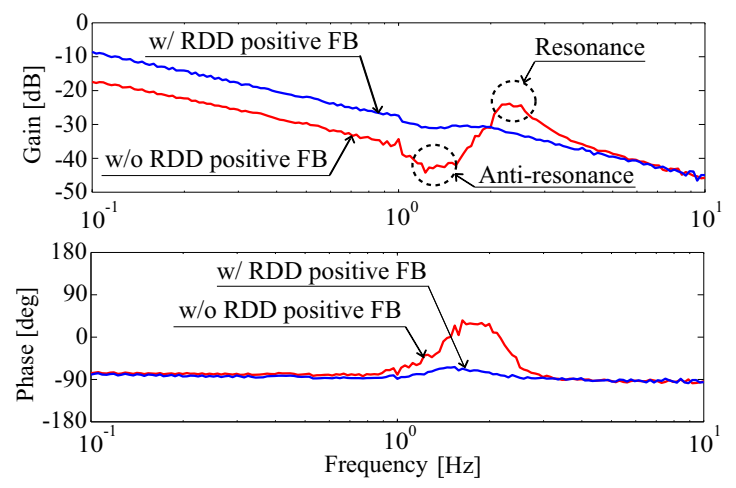

Fig. 5 Elimination of anti-resonance and resonance with RDD positive feedback control (no dead time). By using this control method, the anti-resonance and resonance in the frequency region from 1 to $3 \mathrm{~Hz}$ are suppressed.

\section{Bandwidth expansion in presence of dead time}

\subsection{Drawback of previous RDD positive feedback}

We first show that when the pneumatic AVA has the dead time, the previous RDD positive feedback cannot be implemented. Figure 6 illustrates the block diagram of the control system with the dead time. $T_{d}$ denotes the dead time of the air spring. The transfer function from the output $\tilde{u}$ of pressure PI compensator to the inner pressure $p$ of air spring is given by

$$
\begin{aligned}
\frac{p(s)}{\tilde{u}(s)} & =\frac{G_{q}\left(M s^{2}+D s+K\right) e^{-T_{d} s}}{\left(M s^{2}+D s+K\right)\left(\beta_{0} V_{0} s+c\right)+A_{0}\left(A_{0}-G_{q} k_{t u n} k_{p o s} e^{-T_{d} s}\right) s} \\
& \simeq \frac{G_{q}}{\beta_{0} V_{0} s+c} \frac{\left(M s^{2}+D s+K\right) e^{-T_{d} s}}{M s^{2}+D s+K+K_{\text {air }}\left(1-\frac{G_{q} k_{t u n} k_{p o s} e^{-T_{d} s}}{A_{0}}\right)} .
\end{aligned}
$$


Note that the denominator in (9) and (10) includes the dead time term $e^{-T_{d} s}$. In order to cancel effects of the air spring stiffness, the tunable parameter $k_{t u n}$ of the RDD positive feedback control should be selected as follows:

$$
k_{t u n}=\frac{A_{0}}{G_{q} k_{p o s} e^{-T_{d} s}} .
$$

From Fig. 6, the input voltage $u$ to the servo valve is expressed as

$$
u(s)=\tilde{u}(s)+s k_{t u n} k_{p o s} x(s)
$$

Substituting (11) into (12), we obtain

$$
u(s)=\tilde{u}(s)+\frac{A_{0}}{G_{q}} e^{T_{d} s} s x(s) .
$$

By inverse Laplace transform, we have

$$
u(t)=\tilde{u}(t)+\frac{A_{0}}{G_{q}} \dot{x}\left(t+T_{d}\right)
$$

To determine the input voltage $u$ at time $t$, it is required to know the future value of the displacement derivative, i.e., $\dot{x}\left(t+T_{d}\right)$, a priori. However, this a priori information is unavailable due to the causality of the control system. For this reason, the previous RDD positive feedback control cannot be employed in the presence of the dead time.

Figure 7 shows Bode plots of the transfer function from the reference $r_{p}$ of pressure control system to the output $\tilde{p}$ of pressure feedback gain. This is the result in the case where the dead time exists. The dead time is $T_{d}=0.01 \mathrm{~s}$. As mentioned in the above, since the previous RDD positive feedback control cannot be implemented in the presence of the dead time, this feedback control scheme is not employed in the experiment of Fig. 7. From this figure, we can see that even if the gain $k_{p r}$ of pressure PI compensator becomes large, the anti-resonance and resonance still remain. Moreover, the resonance peak increases with the gain $k_{p r}$ of pressure PI compensator. This causes the destabilization of the control system. Thus, even in the case where the dead time exists, the anti-resonance and resonance have to be eliminated so as to expand the bandwidth.

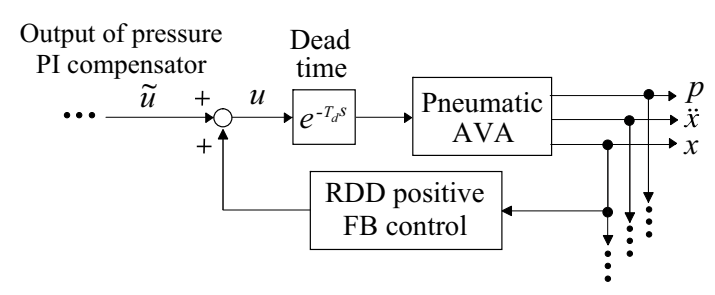

Fig. 6 Block diagram of pneumatic AVA and its control system in presence of dead time. In this figure, $T_{d}$ denotes the dead time of the pneumatic AVA. Due to effects of the dead time, the previous RDD positive feedback cannot be implemented.
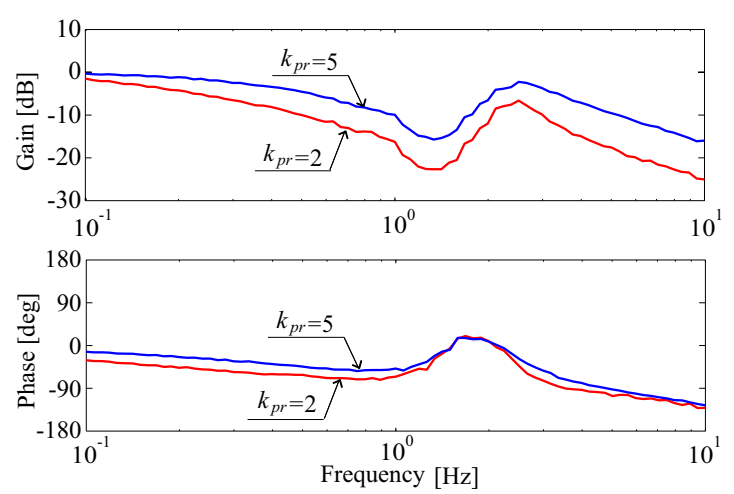

Fig. 7 Bandwidth of pressure control system in presence of dead time (dead time $T_{d}=0.01 \mathrm{~s}$ ). The red and blue lines show the result in the case of $k_{p r}=2$ and $k_{p r}=5$, respectively. The anti-resonance and resonance remain regardless of the gain $k_{p r}$ of pressure PI compensator. Furthermore, when the gain $k_{p r}$ of pressure PI compensator increases, the resonance peak also increases.

\subsection{RDD positive feedback control combined with Smith prediction}

Then, we consider the bandwidth expansion of the pressure feedback control system. Then, we consider the bandwidth expansion of the pressure feedback control system. As mentioned in subsection 3.1, the previous RDD positive feedback control cannot be implemented under the condition where the dead time is present. To compensate effects of the dead time, various control methods have been reported. For instance, as frequency-domain approaches, internal model 


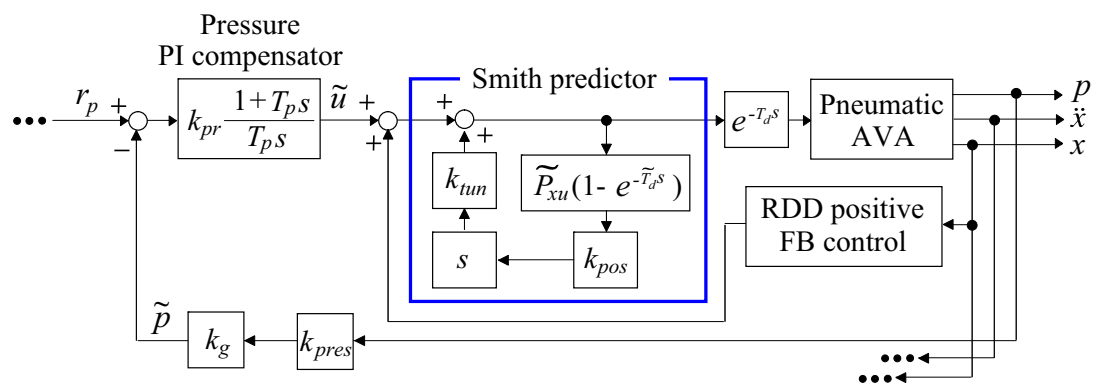

Fig. 8 Block diagram of RDD control scheme combined with Smith predictor approach. In this figure, $\tilde{P}_{x u}$ denotes the model of the pneumatic AVA, and $\tilde{T}_{d}$ denotes the time delay used for Smith prediction. The Smith predictor is employed in the inner loop of the pressure control system so as to compensate effects of the dead time.

control (Morari and Zafiriou, 1989), partial model matching method (Kitamori, 1979; Kitamori, 2001), Smith predictor (Smith, 1959), etc., are widely known. Meanwhile, as time-domain approaches, state-prediction control (Manitius and Olbrot, 1979; Furutani and Araki, 1998), Lyapunov-Krasovskii approach (Fridman and Shaked, 2002), LyapunovRazumikhin approach (Jankovic, 2001), etc., were presented. However, the practical application of these control methods to the dead-time compensation of pneumatic AVA was not sufficiently discussed. On the other hand, we considered the implementation method of the Smith predictor appropriate for the control of pneumatic AVA (Nakamura et al., 2013). In this literature, the model of the pneumatic AVA considering pipe length was presented. The identification method of the dead time was also proposed. Since, from results of Nakamura et al. (2013), the model and dead time of the pneumatic AVA are available, it is expected that the Smith predictor can be implemented and employed for the bandwidth expansion of pressure control system. Moreover, this paper focuses on showing that when the dead-time compensation can be achieved, effects of air spring stiffness $K_{\text {air }}$ are easily cancelled by using previous RDD positive feedback control without other advanced techniques. For these reasons, the Smith prediction approach is adopted. The block diagram of our method is shown in Fig. 8. $\tilde{P}_{x u}$ denotes the model of the pneumatic AVA, and $\tilde{T}_{d}$ denotes the time delay [s] used for Smith prediction. In this method, the RDD positive feedback control is combined with Smith prediction. The transfer function from the output $\tilde{u}$ of pressure PI compensator to the inner pressure $p$ of air spring is expressed as

$$
\begin{aligned}
\frac{p(s)}{\tilde{u}(s)} & =\frac{G_{q}\left(M s^{2}+D s+K\right) e^{-T_{d} s}\left\{1-k_{\text {tun }} k_{p o s} s \tilde{P}_{x u}(s)\left(1-e^{-\tilde{T}_{d} s}\right)\right\}^{-1}}{\left(M s^{2}+D s+K\right)\left(\beta_{0} V_{0} s+c\right)+A_{0}\left[A_{0}-G_{q} k_{t u n} k_{p o s} e^{-T_{d} s}\left\{1-k_{t u n} k_{p o s} s \tilde{P}_{x u}(s)\left(1-e^{-\tilde{T}_{d} s}\right)\right\}^{-1}\right] s} \\
& =\frac{P_{x u}(s)\left(M s^{2}+D s+K\right) e^{-T_{d} s}}{A_{0}\left[1-k_{\text {tun }} k_{p o s} s \tilde{P}_{x u}(s)+k_{t u n} k_{p o s} s\left\{\tilde{P}_{x u}(s) e^{-\tilde{T}_{d} s}-P_{x u}(s) e^{-T_{d} s}\right\}\right]} .
\end{aligned}
$$

By choosing $\tilde{P}_{x u}=P_{x u}$ and $\tilde{T}_{d}=T_{d},(15)$ is rearranged into

$$
\frac{p(s)}{\tilde{u}(s)}=\frac{P_{x u}(s)\left(M s^{2}+D s+K\right) e^{-T_{d} s}}{A_{0}\left\{1-k_{\text {tun }} k_{p o s} s P_{x u}(s)\right\}}
$$

From (1) and (16), we obtain

$$
\frac{p(s)}{\tilde{u}(s)}=\frac{G_{q}\left(M s^{2}+D s+K\right) e^{-T_{d} s}}{\left(M s^{2}+D s+K\right)\left(\beta_{0} V_{0} s+c\right)+A_{0}\left(A_{0}-G_{q} k_{t u n} k_{p o s}\right) s} .
$$

Note that the denominator of (17) is the same as that of (4). As described in subsection 2.3, effects of the air spring stiffness are cancelled by setting the tunable parameter in (17) as $k_{t u n}=A_{0} /\left(G_{q} k_{p o s}\right)$. For this reason, even if the dead time is present, it is not necessary to employ a priori information on the displacement derivative $\dot{x}\left(t+T_{d}\right)$. Hence, one can implement the RDD positive feedback for the pressure control system with the dead time. When the Smith predictor is utilized, the closed-loop transfer function of the pressure control system is expressed as

$$
\begin{aligned}
\frac{p(s)}{r_{p}(s)}= & \frac{G_{q} k_{p r}\left(M s^{2}+D s+K\right)\left(T_{p} s+1\right) e^{-T_{d} s}}{\left(M s^{2}+D s+K\right)\left[T_{p} s\left(\beta_{0} V_{0} s+c\right)\left\{1-k_{\text {tun }} k_{p o s} s \tilde{P}_{x u}(s)\left(1-e^{-\tilde{T}_{d} s}\right)\right\}+G_{q} k_{p r} k_{g} k_{p r e s}\left(T_{p} s+1\right) e^{-T_{d} s}\right]} \\
& \frac{+A_{0} T_{p} s^{2}\left[A_{0}\left\{1-k_{\text {tun }} k_{p o s} s \tilde{P}_{x u}(s)\left(1-e^{-\tilde{T}_{d} s}\right)\right\}-G_{q} k_{t u n} k_{p o s} e^{-T_{d} s}\right]}{}
\end{aligned}
$$


The model and time delay used for Smith prediction are chosen as $\tilde{P}_{x u}=P_{x u}$ and $\tilde{T}_{d}=T_{d}$, respectively. Then, using (5), we can rewrite (18) as

$$
\frac{p(s)}{r_{p}(s)}=\frac{G_{q} k_{p r}\left(T_{p} s+1\right) e^{-T_{d} s}}{T_{p} s\left(\beta_{0} V_{0} s+c\right)+G_{q} k_{p r} k_{g} k_{p r e s}\left(T_{p} s+1\right) e^{-T_{d} s}} .
$$

By setting the time constant of pressure PI compensator as that of air spring (i.e., $T_{p}=T_{\text {air }}$ ), we obtain

$$
\frac{p(s)}{r_{p}(s)}=\frac{1}{k_{g} k_{\text {pres }}} \frac{e^{-T_{d} s}}{\frac{T_{p} c}{G_{q} k_{g} k_{\text {pres }} k_{p r}} s+e^{-T_{d} s}} .
$$

When the gain $k_{p r}$ of the pressure PI compensator is sufficiently large, (20) is approximated as

$$
\frac{p(s)}{r_{p}(s)} \simeq \frac{1}{k_{g} k_{\text {pres }}}=\text { const. }
$$

Hence, the bandwidth expansion of the pressure control system can be achieved by means of our approach.

\subsection{Experimental validation}

Finally, we conduct experiments on the frequency and time responses of the control system for the pneumatic AVA in the presence of the dead time. In the experiments, the values of model parameters used in the Smith predictor are the same as nominal values, which are shown in Table 1. The values of controller parameters and sensor sensitivities are summarized in Table 2. As mentioned in Section 1, the servo valve is set apart from the pneumatic AVA used for the lithography equipment. In semiconductor manufacturing industries, the pipe with length of several ten meters is employed. Due to this, it is necessary to prepare a long pipe in experiment. However, when the pipe is changed for experiments, it leads to serious damage to the connection part of the pipe and air spring. Furthermore, in the room where the pneumatic AVA is set, the floor slopes slightly. Under these experimental conditions, effects of ground vibration are larger than those of the dead time. As a result, there is a difficulty in accurately evaluating effects of dead-time compensation. For these reasons, the dead time is generated in the DSP. The value of the dead time is $T_{d}=0.01 \mathrm{~s}$. The other conditions of experiments in this section are the same as those in subsection 2.3. Under the setting described above, the performance of our approach is evaluated.

\subsubsection{Suppression of anti-resonance and resonance}

Figure 9 shows Bode plots of the transfer function from the output $\tilde{u}$ of pressure PI compensator to the output $k_{\text {pres }} p$ of pressure sensor. From this figure, we can see that when the RDD positive feedback control is used, the anti-resonance and resonance are not observed. In the case with the previous RDD positive feedback control, a priori information on the displacement derivative has to be known (see (11) and (14)). On the other hand, in the case with the proposed approach, one can select the tunable parameter $k_{\text {tun }}$ such that effects of air spring stiffness $K_{\text {air }}$ are cancelled even if a priori information is not available (see (17)). For this reason, the elimination of the anti-resonance and resonance can be achieved in the presence of the dead time. Moreover, by comparing Fig. 9 with Fig. 5, we can also see that when the dead time exists, the phase of Bode plots decreases in the frequency region at about $10 \mathrm{~Hz}$. This phase lag is due to effects of the dead time term $e^{-T_{d} s}$ in (17).

\subsubsection{Bandwidth expansion of pressure control system}

Figure 10 shows Bode plots of the transfer function from the reference $r_{p}$ of pressure control system to the output $\tilde{p}$ of pressure feedback gain. This is the case with the proposed approach (i.e., RDD positive feedback control combined with Smith prediction). From Fig. 10, we can find that the bandwidth of the pressure control system increases with the gain $k_{p r}$ of the pressure PI compensator. This is because when the gain $k_{p r}$ of the pressure PI compensator is large, the gain of the closed-loop transfer function $p / r_{p}$ can be approximated as a constant (see (21)). Hence, in the presence of the dead time, the bandwidth expansion of the pressure control system can be achieved by using our approach.

Moreover, to evaluate the performance of our approach in time domain, we measure the inner pressure $p$ of the air spring. Figure 11(a) shows the step response of the control system in the case without the proposed method. On the other 

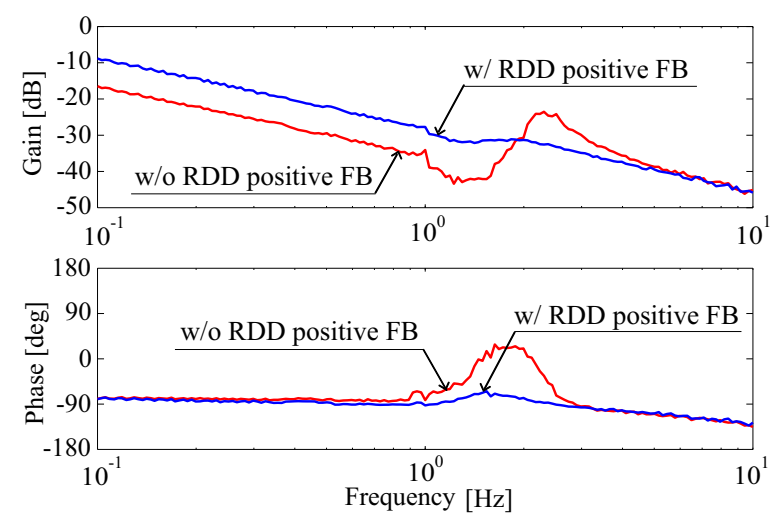

Fig. 9 Elimination of anti-resonance and resonance with RDD positive feedback control (dead time $T_{d}=0.01 \mathrm{~s}$ ). The red and blue lines show the frequency characteristics in the case without and with the RDD positive feedback control. Since effects of the dead time are compensated due to the use of the Smith predictor, the anti-resonance and resonance can be suppressed by using the RDD positive feedback control.

hand, Fig. 11(b) shows the step response in the case with the proposed method. In these figures, the inner pressure at the equilibrium position of the isolation table is set to zero. The step input used as a reference signal is applied to the control system at $t=0.5 \mathrm{~s}$. From Fig. 11(a), we can see that large overshoot and long settling time are observed in the case without the proposed method. As shown in Fig. 7, when the proposed method is not employed, the bandwidth of the pressure control system cannot be sufficiently expanded due to the anti-resonance and resonance. For this reason, the overshoot and settling time become large. On the other hand, from Fig. 11(b), we can find that the rise time of the step responses is improved due to the bandwidth expansion (see responses for $0.5 \leq t \leq 1 \mathrm{~s}$ ). For the same reason, the settling time is also improved. The results shown in Fig. 11(b) indicate that the fast response of the pressure control system is obtained. Therefore, in the presence of the dead time, our proposed approach is effective for the pressure control of the pneumatic AVA.
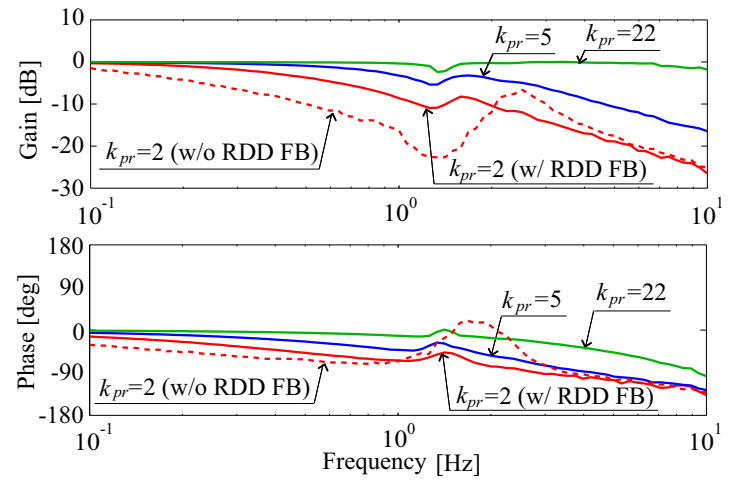

Fig. 10 Bandwidth of pressure control system (dead time $T_{d}=$ $0.01 \mathrm{~s})$. The dashed line and the solid lines show the result in the case without and with the RDD positive feedback control, respectively. The gain $k_{p r}$ of pressure PI compensator is set to 2, 5, and 22 . When the gain $k_{p r}$ becomes large, the bandwidth of the pressure control system expands.

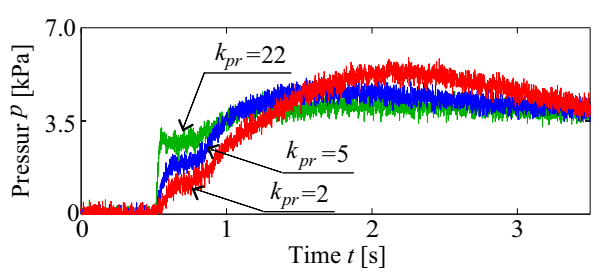

(a) Inner pressure $p$ of air spring (w/o proposed method).

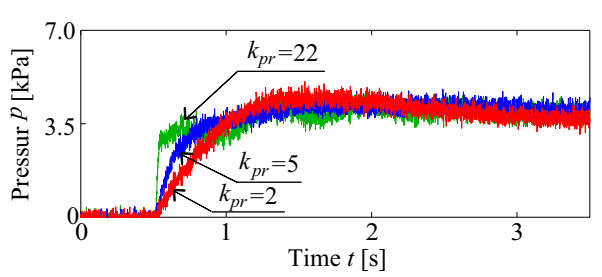

(b) Inner pressure $p$ of air spring (w/ proposed method).

Fig. 11 Step response of control system (dead time $T_{d}=0.01 \mathrm{~s}$ ). The red, blue, and green lines show the time response in the case of $k_{p r}=2,5$, and 22, respectively. The upper and lower figures are the case without and with the RDD positive feedback control combined with Smith prediction. Since the bandwidth of the pressure control system is expanded by means of the proposed method, the rise time and the settling time are improved.

\section{Vibration transmissibility of pneumatic AVA in presence of dead time}

In previous sections, the displacement $x_{0}$ of the floor is assumed to be zero due to the simplicity of discussion. In this section, we investigate effects of floor vibration on the pneumatic AVA in the presence of the dead time. As a performance 
metric for the isolation of the floor vibration, the vibration transmissibility is widely employed (ASML Inc., 2014; Nikon Corp., 2012; Ryaboy, 2014). This metric is defined as the ratio of the displacement amplitude of isolation table to the displacement amplitude of floor, $x / x_{0}$. When the magnitude $\left|x / x_{0}\right|$ is smaller than one (i.e., $0 \mathrm{~dB}$ ), the vibration from the floor is attenuated. For this reason, it is desirable to reduce the magnitude of the vibration transmissibility in the development of the pneumatic AVA.

Before presenting results, we explain the vibration transmissibility in the case where the dead time is not present. In order to discuss this case, Wakui and Fushima (2006) adopted root locus, which is one of geometric approaches. On the other hand, we analytically show how the vibration transmissibility is changed by the RDD positive feedback control. In the control system of Fig. 3, the time constant $T_{a}$ of the acceleration PI compensator is taken as

$$
T_{a}=\frac{T_{p} c}{G_{q} k_{p r} k_{g} k_{\text {pres }}} .
$$

Then, from Fig. 3, the vibration transmissibility is given by

$$
\frac{x(s)}{x_{0}(s)}=\frac{T_{a} s\left\{D s+K+K_{a i r}\left(1-\frac{G_{q} k_{t u n} k_{p o s}}{A_{0}}\right) \frac{T_{p} s}{T_{p} s+1} \frac{T_{a} s}{T_{a} s+1}\right\}+K_{d i s}}{T_{a} s\left\{M s^{2}+\left(D+D_{e}\right) s+K+K_{\text {air }}\left(1-\frac{G_{q} k_{t u n} k_{p o s}}{A_{0}}\right) \frac{T_{p} s}{T_{p} s+1} \frac{T_{a} s}{T_{a} s+1}\right\}+K_{d i s}},
$$

where

$$
\begin{aligned}
K_{\text {dis }} & =\frac{A_{0} k_{a r} k_{s} k_{\text {pos }}}{k_{g} k_{\text {pres }}}, \\
D_{e} & =\frac{A_{0} k_{a r} k_{a} k_{a c c}}{T_{a} k_{g} k_{\text {pres }}},
\end{aligned}
$$

and $K_{d i s}$ denotes servo stiffness $[\mathrm{N} / \mathrm{m}]$ due to the displacement feedback control and $D_{e}$ denotes electrical damping coefficient $[\mathrm{N} \cdot \mathrm{s} / \mathrm{m}]$. From (5) and (23), we have

$$
\frac{x(s)}{x_{0}(s)}=\frac{T_{a} s(D s+K)+K_{d i s}}{T_{a} s\left\{M s^{2}+\left(D+D_{e}\right) s+K\right\}+K_{d i s}} .
$$

From (23) and (24), it is found that when effects of the air spring stiffness are cancelled by using the RDD positive feedback, the vibration transmissibility $x / x_{0}$ changes. Figure 12 shows the simulation result on the vibration transmissibility of the control system in the case where the dead time is not present. The conditions of this simulation are the same as those of experiments presented in subsection 3.3. The values of parameters of the pneumatic AVA used for the simulation are listed in Table 1.

From Fig. 12(a), we can see that the frequency region where the magnitude $\left|x / x_{0}\right|$ of the vibration transmissibility is smaller than $0 \mathrm{~dB}$ is expanded by using the RDD positive feedback control. The reason is as follows. The breakpoint frequencies of the first order lag systems $1 /\left(T_{p} s+1\right)$ and $1 /\left(T_{a} s+1\right)$ in $(23)$ are $1 /\left(2 \pi T_{p}\right) \simeq 0.01$ and $1 /\left(2 \pi T_{a}\right) \simeq 0.53 \mathrm{~Hz}$, respectively. On the other hand, from Fig. 12(b), we can find that the resonance frequency of the vibration transmissibility $x / x_{0}$ is about $1.78 \mathrm{~Hz}$, which is larger than the above breakpoint frequencies. Thus, in the frequency region including the resonance frequency, the first order lag systems can be approximated as $1 /\left(T_{p} s+1\right) \simeq 1 /\left(T_{p} s\right)$ and $1 /\left(T_{a} s+1\right) \simeq 1 /\left(T_{a} s\right)$. Moreover, the servo stiffness is $K_{d i s}=\left(A_{0} k_{a r} k_{s} k_{\text {pos }}\right) /\left(k_{g} k_{\text {pres }}\right) \simeq 6066 \mathrm{~N} / \mathrm{m}$, which is much less than the sum of spring constants of the air spring and mechanical system (i.e., $K_{d i s} \ll K+K_{\text {air }}=26071 \mathrm{~N} / \mathrm{m}$ ). Under these approximations, (23) can be expressed as

$$
\frac{x(s)}{x_{0}(s)} \simeq \frac{D s+K+K_{\text {air }}\left(1-\frac{G_{q} k_{t u n} k_{p o s}}{A_{0}}\right)}{M s^{2}+\left(D+D_{e}\right) s+K+K_{\text {air }}\left(1-\frac{G_{q} k_{t u n} k_{p o s}}{A_{0}}\right)} .
$$

Let $f_{p}$ denote the resonance frequency [Hz] of the vibration transmissibility (25). Then, from (25), the resonance frequency is expressed as

$$
f_{p}=\frac{1}{2 \pi} \sqrt{\frac{1}{M}\left\{K+K_{\text {air }}\left(1-\frac{G_{q} k_{t u n} k_{p o s}}{A_{0}}\right)\right\}-\frac{\left(D+D_{e}\right)^{2}}{4 M^{2}}} .
$$




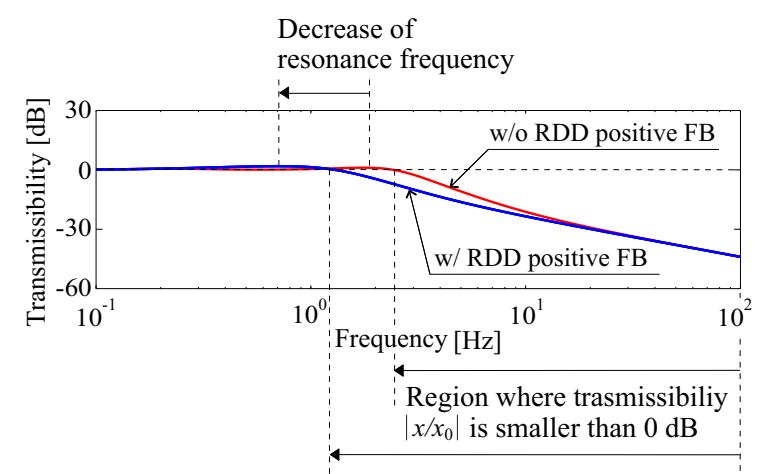

(a) Overview.

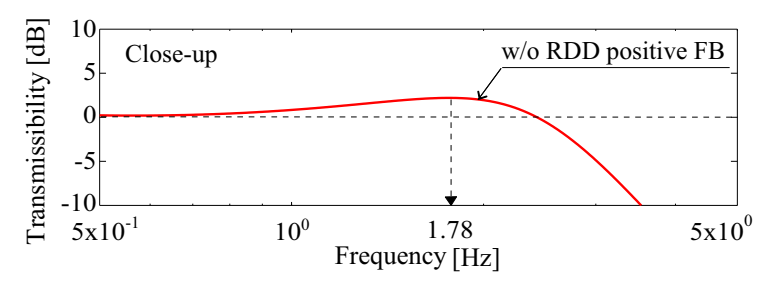

(b) Close-up view.

Fig. 12 Vibration transmissibility $x / x_{0}$ (no dead time). The red and blue lines show the case without and with the RDD positive feedback control scheme. The lower figure is the close-up view of the upper figure. When the RDD positive feedback control is used, effects of air spring stiffness are cancelled. Consequently, the resonance frequency is decreased, resulting in the improvement of the vibration transmissibility.
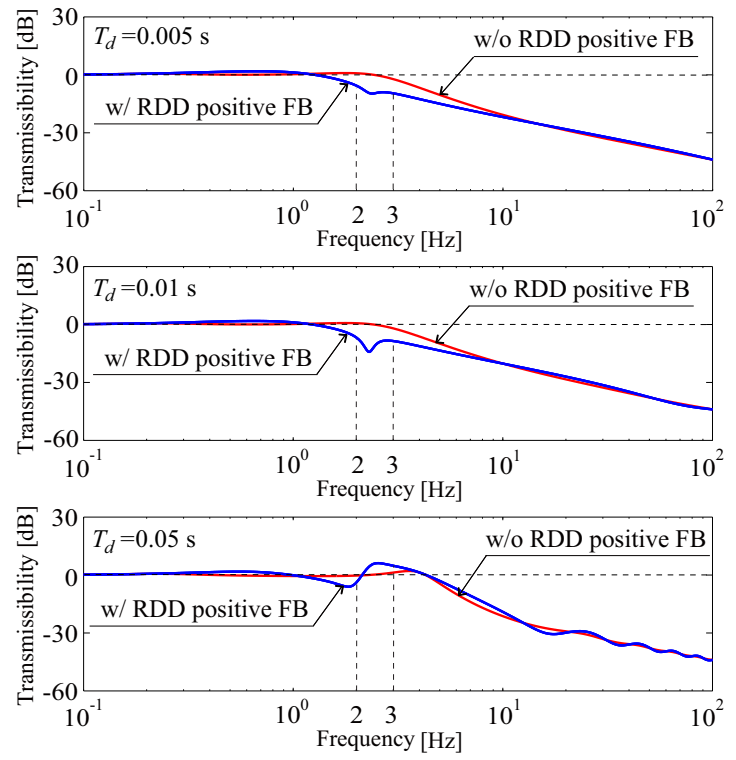

Fig. 13 Vibration transmissibility $x / x_{0}$ (dead time $T_{d}=0.005$, 0.01 , and $0.05 \mathrm{~s}$ ). The red and blue lines show the result in the case without and with the RDD positive feedback control combined with Smith prediction. When the proposed approach is employed, the magnitude $\left|x / x_{0}\right|$ in the case of $T_{d}=0.005$ and $0.01 \mathrm{~s}$ becomes smaller than $0 \mathrm{~dB}$ at about 2 to $3 \mathrm{~Hz}$. Meanwhile, in the case of $T_{d}=0.05 \mathrm{~s}$, the magnitude exceeds $0 \mathrm{~dB}$ at the above frequency region. Due to effects of the dead time, the vibration transmissibility is changed.

Note that (26) for $k_{t u n}=0$ corresponds to the resonance frequency of the vibration transmissibility (25) in the case without the RDD positive feedback control. In this case, the resonance frequency is expressed as

$$
f_{p}=\frac{1}{2 \pi} \sqrt{\frac{K+K_{\text {air }}}{M}-\frac{\left(D+D_{e}\right)^{2}}{4 M^{2}}}, \text { if } k_{\text {tun }}=0 .
$$

In the case with the RDD positive feedback control, from (5) and (26), the resonance frequency is written as

$$
f_{p}=\frac{1}{2 \pi} \sqrt{\frac{K}{M}-\frac{\left(D+D_{e}\right)^{2}}{4 M^{2}}}, \text { if } k_{t u n}=\frac{A_{0}}{G_{q} k_{p o s}} .
$$

Thus, when the dead time is not present, the resonance frequency $f_{p}$ is decreased by using the RDD positive feedback (see (27) and (28)). Consequently, the frequency region where the magnitude $\left|x / x_{0}\right|$ is smaller than $0 \mathrm{~dB}$ is expanded, resulting in the improvement of the vibration transmissibility (see Fig. 12(a)).

Figure 13 shows simulation results of the effects of the dead time $T_{d}$. In this simulation, the dead time is set to $T_{d}=0.005,0.01$, and $0.05 \mathrm{~s}$. The other conditions of Fig. 13 are the same as those of Fig. 12. For the measurement of the vibration transmissibility, it is necessary to generate the floor vibration whose spectrum is rich so as to excite the isolation table. However, due to the structure of the laboratory room, in which the pneumatic AVA is set, the power spectrum of the generated floor vibration is small in low frequency region. For this reason, the vibration transmissibility cannot be accurately evaluated by experiment. Alternatively, we performed the simulation.

From Fig. 13, we can find that when the RDD positive feedback control combined with Smith prediction is employed, the vibration transmissibility at about 2 to $3 \mathrm{~Hz}$ in the case of $T_{d}=0.01 \mathrm{~s}$ is smaller than that in the case of $T_{d}=0.005$ s. Meanwhile, in the case of $T_{d}=0.05 \mathrm{~s}$, the vibration transmissibility exceeds $0 \mathrm{~dB}$ at the above frequency region. This result indicates that effects of isolation of floor vibration change in accordance with the dead time. In the following, we 
explain the detail. From Figs. 3 and 8, the vibration transmissibility in the presence of the dead time is expressed as

$$
\begin{aligned}
\frac{x(s)}{x_{0}(s)}= & \frac{T_{a} s\left[(D s+K)\left\{\left(\beta_{0} V_{0} s+c\right) T_{p} s+G_{q} k_{p r} k_{g} k_{p r e}\left(T_{p} s+1\right) H(s)\right\}+A_{0}\left\{A_{0}-G_{q} k_{t u n} k_{p o s} H(s)\right\} T_{p} s^{2}\right]}{T_{a} s\left[\left(M s^{2}+D s+K\right)\left\{\left(\beta_{0} V_{0} s+c\right) T_{p} s+G_{q} k_{p r} k_{g} k_{p r e}\left(T_{p} s+1\right) H(s)\right\}+A_{0}\left\{A_{0}-G_{q} k_{t u n} k_{p o s} H(s)\right\} T_{p} s^{2}\right]} \\
& \frac{+G_{q} A_{0} k_{p r} k_{a r} k_{s} k_{p o s}\left(T_{p} s+1\right)\left(T_{a} s+1\right) H(s)}{+G_{q} A_{0} k_{p r} k_{a r}\left(T_{p} s+1\right)\left(T_{a} s+1\right)\left(k_{a} k_{a c c} s^{2}+k_{s} k_{p o s}\right) H(s)}
\end{aligned}
$$

where

$$
H(s)=\frac{e^{-T_{d} s}}{1-k_{\text {pos }} k_{t u n} s\left(1-e^{-\tilde{T}_{d} s}\right) \tilde{P}_{x u}(s)} .
$$

The model and time delay used for Smith prediction are taken as $\tilde{P}_{x u}=P_{x u}$ and $\tilde{T}_{d}=T_{d}$. From (1), (5), and (29), we obtain

$$
\frac{x(s)}{x_{0}(s)}=\frac{(D s+K)\left\{T_{p}^{2} c^{2} s^{2} L(s)+G_{q} k_{p r} k_{g} k_{p r e} T_{p} c s e^{-T_{d} s}\right\}+A_{0} G_{q}^{2} k_{p r}^{2} k_{a r} k_{s} k_{p o s} k_{g} k_{p r e}\left(T_{a} s+1\right) e^{-T_{d} s}}{\left(M s^{2}+D s+K\right)\left(T_{p}^{2} c^{2} s^{2}+G_{q} k_{p r} k_{g} k_{p r e} T_{p} c s e^{-T_{d} s}\right)+A_{0} G_{q}^{2} k_{p r}^{2} k_{a r}\left(k_{a} k_{a c c} s^{2}+k_{s} k_{p o s}\right)\left(T_{a} s+1\right) e^{-T_{d} s}}
$$

where

$$
L(s)=\frac{\left(M s^{2}+D s+K\right)\left(\beta_{0} V_{0} s+c\right)+A_{0}^{2} s e^{-T_{d} s}+\left\{\left(M s^{2}+D s+K\right) A_{0}^{2} s /(D s+K)\right\}\left(1-e^{-T_{d} s}\right)}{\left(M s^{2}+D s+K\right)\left(\beta_{0} V_{0} s+c\right)+A_{0}^{2} s} .
$$

From (22) and (30), we have

$$
\frac{x(s)}{x_{0}(s)}=\frac{T_{a} s(D s+K)\left(T_{a} s L(s)+e^{-T_{d} s}\right)+K_{d i s}\left(T_{a} s+1\right) e^{-T_{d} s}}{T_{a} s\left(M s^{2}+D s+K\right)\left(T_{a} s+e^{-T_{d} s}\right)+\left(T_{a} D_{e} s^{2}+K_{d i s}\right)\left(T_{a} s+1\right) e^{-T_{d} s}},
$$

Let

$$
\begin{aligned}
& N_{1}(s)=\frac{T_{a} s(D s+K)+K_{d i s}}{T_{a} s\left\{M s^{2}+\left(D+D_{e}\right) s+K\right\}+K_{d i s}} \\
& N_{2}(s)=\frac{e^{-T_{d} s}+T_{a} s\left\{\frac{T_{a} s(D s+K) L(s)+K_{d i s} e^{-T_{d} s}}{T_{a} s(D s+K)+K_{d i s}}\right\}}{e^{-T_{d} s}+T_{a} s\left[\frac{T_{a} s\left\{M s^{2}+\left(D+D_{e} e^{-T_{d} s}\right) s+K\right\}+K_{d i s} e^{-T_{d} s}}{T_{a} s\left\{M s^{2}+\left(D+D_{e}\right) s+K\right\}+K_{d i s}}\right]} .
\end{aligned}
$$

Then, the vibration transmissibility (31) is expressed as

$$
\frac{x(s)}{x_{0}(s)}=N_{1}(s) N_{2}(s)
$$

Note that $N_{1}(s)$ corresponds to (24), i.e., the vibration transmissibility in the case where the dead time is not present. On the other hand, $N_{2}(s)$ includes the dead time term $e^{-T_{d} s}$. For this reason, in the presence of the dead time, the vibration transmissibility is changed due to effects of $N_{2}(s)$ in (34), where the dead time term is included. Therefore, when the dead time exists, there is trade-off between the improvement of vibration transmissibility and the bandwidth expansion of pressure control system.

\section{Conclusion}

In this paper, we considered the bandwidth expansion of the pressure control system with the dead time of the air spring. To extend the control bandwidth, the anti-resonance and resonance in the pneumatic system are eliminated by means of the RDD positive feedback control combined with the Smith prediction. The advantage of our method is that the RDD positive feedback control can be implemented without a priori information on the displacement derivative. From experimental results, we found that when the proposed method is employed in the presence of the dead time, the control bandwidth can be increased by tuning the gain $k_{p r}$ of the pressure PI compensator. Moreover, we also found that due to the dead time, the vibration transmissibility increases in a certain frequency region. Our future work is to achieve the bandwidth expansion considering the improvement of vibration transmissibility. 


\section{Acknowledgement}

This work is supported in part by JSPS KAKENHI, Grant-in-Aid for Scientific Research (C) Grant Number 25420178.

\section{References}

ASML Inc., Lithographic apparatus comprising a vibration isolation support device, United States patent disclosure US8102505B2 (2012)

Butler, H., Position Control in lithographic equipment; an enabler for current-day chip manufacturing, IEEE Control Systems Magazine, Vol.31, No.5 (2011) pp.28-47.

Canon Inc., Anti-vibration apparatus and exposure apparatus using the same, United States patent disclosure US6213443B1 (2001a)

Canon Inc., Active anti-vibration apparatus and exposure apparatus, European patent disclosure EP1124078A2 (2001b)

Fridman, E. and Shaked, U., A descriptor system approach to $H_{\infty}$ control of linear time-delay systems, IEEE Transactions on Automatic Control, Vol.47, No.2 (2002), pp.253-270.

Furutani, E. and Araki, M., Robust stability of state-predictive and Smith control systems for plants with a pure delay, International Journal of Robust and Nonlinear Control, Vol.8, No.10 (1998), pp.907-919.

Heertjes, M. F., Sahin, I. H., van de Wouw, N. and Heemels, W. P. M. H., Switching control in vibration isolation systems, IEEE Transactions on Control Systems Technology, Vol.21, No.3 (2013) pp.626-635.

Jankovic, M., Control Lyapunov-Razumikhin functions and robust stabilization of time delay systems, IEEE Transactions on Automatic Control, Vol.46, No.7 (2001), pp.1048-1060.

Kawashima, K., Kato T., Sawamoto, K. and Kagawa, T., Realization of virtual sub chamber on active controlled pneumatic isolation table with pressure differentiator, Vol.31, No.2 (2007) pp.139-145.

Kitamori, T., Partial model matching method conformable to physical and engineering actualities, Proceedings of the IFAC Symposium on System Structure and Control (2001), pp.141-146.

Kitamori T., A method of control system design based upon partial knowledge about controlled processes, Transactions of the Society of Instrument and Control Engineers, Vol.15, No.4 (1979), pp.549-555. (in Japanese)

Lee, J. H. and Kim, K. J., Modeling of nonlinear complex stiffness of dual-chamber pneumatic spring for precision vibration isolations, Journal of Sound and Vibration, Vol.301, No.3-5 (2007) pp.909-926.

Manitius, A. and Olbrot, A. W., Finite spectrum assignment problem for systems with delays, IEEE Transactions on Automatic Control, Vol.24, No.4 (1979), pp.541-552.

Morari, M. and Zafiriou, E., Robust Process Control (1989), pp.75-84, Prentice-Hall, Inc.

Nakamura, Y., Goto, S. and Wakui, S., Tuning methods of a Smith predictor for pneumatic active anti-vibration apparatuses, Journal of Advanced Mechanical Design, Systems, and Manufacturing, Vol.7, No.4 (2013) pp.666-676.

Nikon Corp., Stage assembly with lightweight fine stage and low transmissibility, United States patent disclosure US7869000B2 (2011)

$\mathrm{Pu}, \mathrm{H}$., Luo, X. and Chen, X., Modeling and analysis of dual-chamber pneumatic spring with adjustable damping for precision vibration isolation, Journal of Sound and Vibration, Vol.330, No.15 (2011) pp.3578-3590.

Ryaboy, V. M., Static and dynamic stability of pneumatic vibration isolators and systems of isolators, Journal of Sound and Vibration, Vol.333, No.1 (2014) pp.31-51.

Shin, Y. H. and Kim, K. J., Performance enhancement of pneumatic vibration isolation tables in low frequency range by time delay control, Journal of Sound and Vibration, Vol.321, No.3-5 (2009) pp.537-553.

Shin, Y. H., Kim, K. J., Chang, P. H. and Han, D. K., Three degrees of freedom active control of pneumatic vibration isolation table by pneumatic and time delay control technique, Transactions of ASME, Journal of Vibration and Acoustics, Vol.132, No.5 (2010), pp.1-12.

Smith, O. J. M., A controller to overcome dead time, ISA Journal, Vol.6, No.2 (1959) pp.28-33.

Wakui, S., Incline compensation control using an air-spring type active isolated apparatus, Precision Engineering Vol.27, No.2 (2003) pp. 170-174.

Wakui, S. and Fushima C., Wide frequency band of pressure feedback using positive feedback for an air spring type antivibration apparatus, Transactions of the Japan Society of Mechanical Engineers, Series C, Vol.72, No.719 (2006) pp.2219-2225. (in Japanese) 